\title{
ON THE STRUCTURE OF CLOSED IDEALS
}

\author{
JOSEPH P. BRENNAN and WOLMER V. VASCONCELOS*
}

\section{Introduction}

Let $R$ be a Noetherian ring for which we seek to map a path to its integral closure. The ring will be specified in some manner, say by generators and relations over a field or over $Z$.

The multiplier approach consists in the following. For each ideal $I$ containing regular elements, the ring of endomorphisms of $I, \operatorname{Hom}_{R}(I, I)$, can be identified as an integral extension of $R$ in its total ring of fractions. We say that $I$ is closed if $R=\operatorname{Hom}_{R}(I, I)$. Despite the arithmetical definition, this notion will be better expressed by homological means.

Examples of closed ideals are principal ideals, or more generally invertible ideals, canonical modules of rings satisfying Serre's condition $S_{2}$, and ideals of grade at least two. The condition holds universally for all nonzero ideals of $R$ precisely when $R$ is integrally closed. It is in connection with this last fact that our interest in non-closed ideals lies: Finding the integral closure $\bar{R}$ of $R$ amounts to identifying sufficiently many non-closed ideals $I_{\alpha}$ such that

$$
\bar{R}=\bigcup_{\alpha} \operatorname{Hom}_{R}\left(I_{\alpha}, I_{\alpha}\right)
$$

In [12] for affine domains, Jacobian ideals were systematically used in order to enlarge the ring into its integral closure. One of our motivations is to lift the dependency on the characteristic by identifying other classes of ideals with the requisite property. As a result one obtains an algorithmic path to the integral closure for algebras over any field or even Z-algebras.

An alternative way to construct the integral closure is to form blow up rings. If $I$ is some ideal, the directed union

$$
R^{(I)}=\bigcup_{n} \operatorname{Hom}_{R}\left(I^{n}, I^{n}\right)
$$

produces an integral extension of $R$ with the property that in dimension 1 the

\footnotetext{
* Partially supported by the NSF.

Received July 15, 1997; in revised form March 23, 1998.
} 
ideal $I \cdot R^{(I)}$ is invertible. When $\operatorname{dim} R=1$ there is a large body of literature on this process (see [9]).

Although our interest is on non-closed (critical?) ideals, the overall approach is to provide means to demark the set of closed ideals from the nonclosed ones. It is clear that if $I$ is an ideal and $J$ is an invertible ideal then $I$ is closed if and only if $J I$ is closed. The equivalence classes of closed ideals under this action will be denoted by $\operatorname{Clos}(R)$.

Three main questions are:

- What are the closed ideals? In particular, which ideals cannot be closed?

- Are there bounds for the number of generators of closed ideals of codimension 1 which are Cohen-Macaulay?

- What is the structure of $\operatorname{Clos}(R)$ ? Which operations can be defined on $\operatorname{Clos}(R)$ and when is this set finite?

We will give partial answers to each of these. We shall now describe our results.

\section{Closed ideals and the canonical module}

Throughout $R$ will be a Noetherian ring admitting a canonical module $\omega_{R}=\omega$ whose total ring of fractions $Q$ is Artinian. Most of the constructions given take place in $Q$. As usual, we say that an $R$-module $E$ is a torsion module if $E \otimes_{R} Q=0$.

We begin by giving a general criterion for closedness of ideals. We refer to [2] for basic facts about canonical modules. We define the $I^{\omega}$ the $\omega$-dual of the ideal I, to be

$$
I^{\omega}=\operatorname{Hom}_{R}(I, \omega) .
$$

We say that the ideal $I$ is $\omega$-reflexive if the canonical mapping

$$
\left.I \longrightarrow \operatorname{Hom}_{R}\left(\operatorname{Hom}_{R}(I, \omega), \omega\right) \quad \text { (i.e. } I \longrightarrow\left(I^{\omega}\right)^{\omega}\right)
$$

is an isomorphism. We note that if $\operatorname{dim} R=1$ then all ideals are $\omega$-reflexive. We reserve the terminology divisorial for those ideals for which $I=\operatorname{Hom}_{R}\left(\operatorname{Hom}_{R}(I, R), R\right)$.

Proposition 2.1. Let $R$ be a Noetherian ring satisfying Serre's condition $S_{2}$ that has a canonical module $\omega$. A nonzero $\omega$-reflexive ideal $I$ is closed if and only if the evaluation mapping

$$
I \otimes_{R} \operatorname{Hom}_{R}(I, \omega) \stackrel{\mathrm{ev}_{I}}{\longrightarrow} \omega,
$$

given by $\operatorname{ev}_{I}(x \otimes \alpha)=\alpha(x)$, has a cokernel of codimension at least 2 . 
Proof. Denote by $K$ and $C$, respectively, the kernel and cokernel of $\mathrm{ev}_{I}$; note that these are torsion modules. We have exact sequences

$$
0 \rightarrow K \longrightarrow I \otimes_{R} \operatorname{Hom}_{R}(I, \omega) \longrightarrow T \rightarrow 0
$$

and

$$
0 \rightarrow T \longrightarrow \omega \longrightarrow C \rightarrow 0
$$

where $T$ is the image of $\operatorname{ev}_{I}$. Applying $\operatorname{Hom}_{R}(\cdot, \omega)$ to both sequences, we get an exact sequence

$$
\begin{aligned}
R & =\operatorname{Hom}_{R}(\omega, \omega) \hookrightarrow \operatorname{Hom}_{R}\left(I \otimes \operatorname{Hom}_{R}(I, \omega), \omega\right) \\
& =\operatorname{Hom}\left(I, \operatorname{Hom}_{R}\left(\operatorname{Hom}_{R}(I, \omega), \omega\right)\right)=\operatorname{Hom}_{R}(I, I) \longrightarrow \operatorname{Ext}_{R}^{1}(C, \omega) \rightarrow 0 .
\end{aligned}
$$

Since both $R$ and $\operatorname{Hom}_{R}\left(I \otimes \operatorname{Hom}_{R}(I, \omega), \omega\right)$ are modules with the condition $S_{2}$, to prove that they are equal it suffices to verify equality at each localization $R_{\mathfrak{p}}$ of dimension 1 . This is precisely the condition on $C$.

\section{Dimension one}

Because of the reduction aforded by the preceeding proposition, we shall focus henceforth on rings of dimension 1. Let us rephrase the characterization given above into a more direct criterion.

Proposition 3.1. Let $R$ be a Cohen-Macaulay ring of dimension 1 , with a canonical ideal $\omega$. A nonzero fractionary ideal $I$ is closed if and only if it is $\omega-$ invertible, that is

$$
I \cdot \operatorname{Hom}_{R}(I, \omega)=\omega .
$$

COROLlary 3.2. If $R$ is a Gorenstein ring of dimension 1 then every closed ideal is invertable.

We give a companion criterion of closedness in terms of the faithful modules of $R /(x)$ for any parameter $x$. Set $\bar{\omega}$ for the canonical module of $R /(x)$, we will use the notation $\bar{\omega}=\omega / x \omega$ and $\bar{R}=R / x$.

Proposition 3.3. Let $(R, \mathfrak{m})$ be a Cohen-Macaulay local ring of dimension 1 and let $I$ be an $\mathfrak{m}$-primary ideal. Then $I$ is closed if and only if for any parameter $x$ the module $I / x I$ is faithful over $R /(x)$.

Proof. The proposition will follow from the two following observations. First, a finitely generated module $E$ over an Artin local ring $A$ is faithful when the evaluation mapping 


$$
E \otimes_{A} \operatorname{Hom}_{A}\left(E, \omega_{A}\right) \longrightarrow \omega_{A}
$$

is surjective. Second, for a torsionfree module $M$ of $R$,

$$
\operatorname{Hom}_{R}(I, \omega) \otimes \bar{R}=\operatorname{Hom}_{\bar{R}}(I / x I, \bar{\omega})
$$

For an $\mathfrak{m}$-primary ideal $I$, the mapping

$$
I \otimes_{R} \operatorname{Hom}_{R}(I, \omega) \longrightarrow \omega
$$

is surjective if and only if for a parameter $x$ of $R$, tensoring with $R /(x)$, we get another surjection

$$
I / x I \otimes_{R} \operatorname{Hom}_{R}(I, \omega) \otimes R /(x) \longrightarrow \omega / x \omega \rightarrow 0 .
$$

It follows that $I$ is closed if and only if $I / x I$ is $R /(x)$-faithful.

For instance, if $I$ is generated by the regular elements $x$ and $y$, the condition above is equivalent to the equality $x I \cap y I=x y I$.

The $\omega$-involution and square roots

The process of taking the $\omega$-dual of a module, defines an involution on the closed ideals.

Proposition 3.4. Let $\omega$ be a canonical ideal of $R$. Then the correspondence

$$
\alpha:[I] \mapsto\left[I^{\omega}\right]
$$

defines an involution on $\operatorname{Clos}(R)$. Its fixed points are the classes of ideals $[I]$ such that $I^{2} \simeq \omega$.

The proof follows immediately from proposition 2.2. Note that there is relationship between the number of generators of $I$ and the Cohen-Macaulay (locally) type of $R$ for the fixed points of the involution:

$$
\operatorname{type}(R)=\nu(\omega)=\nu\left(I^{2}\right) \leq\left(\begin{array}{c}
\nu(I)+1 \\
2
\end{array}\right) .
$$

ExAmple 3.5. Here is a closed ideal $I$ that satisfies $I^{2} \simeq \omega$. Let $R=$ $k\left[\left[t^{4}, t^{5}, t^{6}, t^{7}\right]\right]$ and let $I=\left(t^{4}, t^{5}\right)$. The isomorphism class of the canonical module has as a representative the ideal $\omega=\left(t^{4}, t^{5}, t^{6}\right)$. Thus $I^{2}=$ $\left(t^{8}, t^{9}, t^{10}\right)=t^{4} \omega$ is also in the isomorphism class of the canonical module.

REMARK 3.6. The 'roots' of $\omega$ can be used to define Gorenstein algebras. Let us indicate how this is done for the square roots. Let $I$ be an ideal such that $I^{2} \simeq \omega$. We interpret this isomorphism as a perfect pairing

$$
I \otimes I \longrightarrow \omega .
$$

Let $A$ be the algebra 


$$
A=R \oplus I \oplus \omega
$$

which we view as graded in degrees $0,1,2$ (in particular $I \cdot \omega=0$ ). It is straightforward to verify that $A$ is a 1 -dimensional Gorenstein ring.

Proposition 3.7. Let $R$ be a local domain of dimension 1 and multiplicity $\operatorname{deg} R=e$. If the reduction number of $\omega$ is larger than $\left\lceil\frac{e-1}{2}\right\rceil$ then $\alpha$ has no fixed points.

Proof. We recall that the reduction number $\operatorname{red}(I)$ of an ideal $I$ is the smallest integer $r$ for which $I^{r}$ is an invertible ideal of the blowup $\operatorname{ring} R^{(I)}$. By passing over a ring with infinite residue field, this is equivalent to saying that $I^{r+1}=x I^{r}$, for some $x \in I$. For all ideals, $\operatorname{red}(I) \leq e-1$.

If $r=2 s \leq e-1$, we have

$$
\omega^{s+1}=I^{r+2}=x^{2} I^{2 s}=x^{2} \omega^{s},
$$

which is a contradiction. The other case, $r=2 s+1$, is also impossible.

On the other hand, the canonical module provides a collection of nonclosed ideals.

Proposition 3.8. Let $\omega$ be a canonical ideal of $R$. If $R$ is not Gorenstein, then no power $\omega^{n}, n \geq 2$, is closed.

Proof. We consider the case $n=2$, the others are similar. Denote by $L$ the fractionary ideal $\operatorname{Hom}_{R}\left(\omega^{2}, \omega\right)$. By assumption, $L \cdot \omega^{2}=\omega$. On the other hand, note that from

$$
L^{2} \cdot \omega^{3}=L \cdot \omega^{2} \cdot \omega=L \cdot \omega \cdot \omega=\omega,
$$

we get $L^{2} \subset \operatorname{Hom}_{R}\left(\omega^{3}, \omega\right)$ and then applying the Proposition obtain that $\omega^{3}$ is closed as well. Similarly it will follow that all powers of $\omega$ are closed. This implies that the blowup ring $R^{(\omega)}=R$, a contradiction since $R$ is not Gorenstein.

\section{Irreducible ideals}

Proposition 3.9. Let $R$ be a Cohen-Macaulay ring of type s and let $I$ be an ideal and suppose that $R / I$ has Cohen-Macaulay type $r$. Then $I^{\omega}$ is generated by $r$ elements. If I is an irreducible closed ideal then it is a canonical module of $R$.

Proof. The assumption means that the canonical module of $R / I$ is generated by $r$ elements. Consider the exact sequence

$$
0 \rightarrow I \longrightarrow R \longrightarrow R / I \rightarrow 0,
$$

and map it into $\omega$. The exactness of 


$$
0 \rightarrow \operatorname{Hom}_{R}(R, \omega) \longrightarrow \operatorname{Hom}_{R}(I, \omega) \longrightarrow \operatorname{Ext}_{R}^{1}(R / I, \omega)=\omega_{R / I} \rightarrow 0
$$

means that $\operatorname{Hom}_{R}(I, \omega)$ is generated by multiplication by the elements of $\omega$ plus $\alpha_{1}, \ldots, \alpha_{r}$, one $\alpha_{i}$ for each generator of $\operatorname{Ext}_{R}(R / I, \omega)$.

Since $I$ is closed, we must have that

$$
\operatorname{Hom}_{R}(I, \omega) \cdot I=\omega \cdot I+\sum_{i=1}^{r} \alpha_{i}(I)=\omega .
$$

By the Nakayama lemma it follows that $\sum_{i=1}^{r} \alpha_{i}(I)=\omega$. Any $\beta \in I^{\omega}$ can thus be written as

$$
\beta(x)=a \cdot x+\sum_{i=1}^{r} p_{i} \alpha_{i}(x), \quad x \in I,
$$

where $a \in \omega$ depends only on $\beta$. From the trace equality above, we may write $a$ as

$$
a=\sum_{i=1}^{r} s_{i} \alpha_{i}\left(a_{i}\right)
$$

from which we get

$$
\begin{aligned}
\beta(x) & =\left(\sum_{i=1}^{r} s_{i} \alpha_{i}\left(a_{i}\right)\right) x+\sum_{i=1}^{r} p_{i} \alpha_{i}(x) \\
& =\sum_{i=1}^{r}\left(p_{i}+s_{i} a_{i}\right) \alpha_{i}(x),
\end{aligned}
$$

since for any $\alpha \in I^{\omega}$, and $x, y \in I$,

$$
\alpha(x y)=x \alpha(y)=y \alpha(x) .
$$

When $r=1$ this implies that $I \simeq \omega$.

REMARK 3.10. The last assertion means that the only closed irreducible ideals of $R$ are $\omega$ or its multiples, $x \cdot \omega$. This is a kind of converse, for rings of dimension one, of a well-known observation of Peskine saying: If $R$ is a Cohen-Macaulay local ring and $\omega$ is one of its canonical ideals then $R / \omega$ is a Gorenstein ring.

We further observe that the number of irreducible components of an arbitrary ideal $I$ is not always the same as that of $x I$. Consider the ring $R=k[x, y] /\left(x^{3}+y^{3}\right)$, and $I=(x, y)$. Then $I^{3}=x I^{2}$, and the number of irreducible components of $I^{2}$ is 2, while that of $I^{3}$ is 3. This implies that the property of being an irreducible ideal is not retained by its isomorphism class: if 


$$
I^{2}=L_{1} \cap L_{2}
$$

is an irreducible decomposition,

$$
x I^{2}=x L_{1} \cap x L_{2}
$$

cannot be an irreducible decomposition and therefore $x L_{1}$ or $x L_{2}$ will not be irreducible.

Thus far we have emphasized $\omega$ as the premier closed ideal. Now we give a construction of rings with other kinds of closed ideals

Let $(R, \mathfrak{m})$ be a Cohen-Macaulay local ring of dimension 1. Let

$$
\varphi=\left[\begin{array}{ccc}
a_{1,1} & \cdots & a_{1, n+1} \\
\vdots & \ddots & \vdots \\
a_{n, 1} & \cdots & a_{n, n+1}
\end{array}\right]
$$

be a matrix with entries in the ring of polynomials $R[x, y]$ and let $L$ be the ideal generated by the minors of size $n$. Suppose that $S=R[x, y] / L$ is an integral extension of $R$. In particular the ideal $\mathrm{L}$ has grade 2 so that by the Hilbert-Burch theorem, we have aexact complex

$$
0 \rightarrow R[x, y]^{n} \stackrel{\varphi}{\longrightarrow} R[x, y]^{n+1} \longrightarrow R[x, y] \longrightarrow S \rightarrow 0 .
$$

To show that $S$ is $R$-flat, by the local criterion of flatness ([10, Theorem 49]), it suffices to show that $\operatorname{Tor}_{1}^{R}(R / \mathfrak{m}, S)=0$. For that, reduce the complex above(which is a free $R$-resolution of $S$ )modulo $\mathfrak{m}$ and observe that the ideal generated by the maximal minors of $\bar{\varphi}$ has height 2 in the $\operatorname{ring} R / \mathfrak{m}[x, y]$ and thus the corresponding Hilbert-Burch complex is exact.

A similar construction can be carried out for a for a $m \times n$ matrix with entries in $R\left[x_{1}, \ldots, x_{d}\right], d=n-m+1$, using the Eagon-Northcott complex instead.

Given a faithfully flat extension $R \mapsto S$, there is an embedding

$$
\psi: \operatorname{Clos}(R) \mapsto \operatorname{Clos}(S), \quad \psi([I])=\left[I \otimes_{R} S\right] .
$$

In particular the ideal $\omega_{R} \otimes_{R} S$ is closed but rarely isomorphic to either $S$ or $\omega_{S}$. Indeed, if all the entries of $\varphi$ are polynomials without constant terms, the $\operatorname{ring} S / \mathfrak{m} S$ has Cohen-Macaulay type $n$ and therefore all localizations of $S$ have Cohen-Macaulay type the product of $n$ with the Cohen-Macaulay type of $R$ (see [6, Theorem1.24]). 


\section{Finiteness and boundedness}

Let $R$ be a Cohen-Macaulay ring of dimension 1 with a finite number of non-Gorenstein singularities

$$
N G(R)=\left\{\wp_{1}, \ldots, \wp_{r}\right\} .
$$

For instance, if $R$ is a reduced affine ring of dimension 1 then this will occur. In fact we have implicitly assumed that $Q$ is Gorenstein when we have taken $\omega$ to be an ideal of $R$.

Proposition 4.1. In this case, there is an embedding

$$
\operatorname{Clos}(R) \hookrightarrow \prod_{1 \leq i \leq r} \operatorname{Clos}\left(R_{\wp_{i}}\right) .
$$

Proof. If $I$ and $J$ are two closed ideals with isomorphic images in all localizations, the module $L=\operatorname{Hom}_{R}(I, J)$ is invertible and is such that $L I=J$.

This helps to focus the question on the following: If $R$ is a 1 -dimensional local domain, when is $\operatorname{Clos}(R)$ finite? The answer in general is negative.

ExAmple 4.2. Let $k$ be an infinite field and let $K$ be a field extension of degree 5. For an indeterminate $x$, the ring $R=k+x K[[x]]$ admits no proper extension between itself and its integral closure $K[[x]]$. It follows that the ideals of $R$ are either closedor $x^{n} K[[x]]$ for some $n$. On the other hand, the ring $R$ is known to have an infinite number of non-isomorphic ideals [14, Proposition 4.2].

One can also explicitly obtain an infinite family of non-isomorphic closed ideals.

EXAmple 4.3. Let $k$ be an infinite field and $R=k\left[\left[t^{4}, t^{5}, t^{6}, t^{7}\right]\right]$. For $\alpha \in k$, let $I_{\alpha}=\left(t^{4}, t^{5}+\alpha t^{6}\right)$. The $\left\{I_{\alpha}\right\}_{\alpha \in k}$ are all closed ideals and $I_{\beta} \cong I_{\gamma}$ only if $\beta=\gamma$.

\section{Bounds on the number of generators}

In this section we present bounds on the number of generators of closed ideals. In particular we investigate whether there is a bound $\nu(I) \leq \operatorname{type}(R)$. Our results establish this in the case that the type of $R$ is small (less than or equal to two) or large (greater than or equal to the multiplicity of the ring minus three).

Gulliksen results. There is a result in [7] on the lengths of faithful modules over Artin local rings that is relevant to our discussion here. It establishes the bound $\nu(I) \leq \operatorname{type}(R)$ when type $(R) \leq 2$.

Let $A$ be a Artin local ring and $M$ a faithful $A$-module of finite length. 
THeORem 4.4. If the Cohen-Macaulay type of $A$ is at most 3, then $\ell(M) \geq \ell(A)$.

It is not difficult to show that as a consequence of our discussion one has:

Corollary 4.5. Let $R$ be a Cohen-Macaulay local ring of dimension one and type two. Then

$$
\operatorname{Clos}(R)=\{[\omega],[R]\} .
$$

Rings of large type. In investigating Artin local rings of large type, we will make use of the notion of Loewy length of a module. Let $M$ be a module of finite length over the Artin local ring $A$ whose maximal ideal we denote by $\mathfrak{m}$. The Loewy length of $M$ is:

$$
\ell \ell(M)=\min \left\{n>\mid>\mathfrak{m}^{n} M=0\right\} .
$$

The Lowey length enters into the discussion through the following observation:

Lemma 4.6. Let $M$ be a finite length module $(\ell(M)<\infty)$ over an Artin local ring with type of $M$ equal to $r$, then $\ell \ell(M)+r \leq \ell(M)+1$.

Also, one sees that when the Loewy length of an Artin local ring is small, that the type of the ring must be large.

Lemma 4.7. Let $A$ be an Artin local ring of length e and maximal ideal $\mathfrak{m}$, and $\ell \ell(A) \leq 2$ then the type of $A$ is equal to $e-\ell \ell(A)+1$.

Proof. If $\ell \ell(A)=1$, then $\mathfrak{m}=0$ and $A$ is a field. Hence the type of $A$ is one, which is the value of $e-\ell \ell(A)+1$.

If $\ell \ell(A)=2$, then $\mathfrak{m}$ is the socle of $A$. Hence the type of $A$ is $e-1$, which is the value of $e-\ell \ell(A)+1$.

We are now in a position to prove the principal result of this section. We denote by $r(R)$ the Cohen-Macaulay type of the ring $R$.

Proposition 4.8. If $R$ is a one dimensional Noetherian local CohenMacaulay ring with $r(R) \geq \operatorname{deg} R-3$ then every closed ideal of $R$ can be generated by at most $r(R)$ generators.

Proof. Let $I$ be a closed ideal of $R$. By Proposition 3.9, for any parameter $x$, the module $M=I / x I$ is a faithful module over the ring $A=R / x$. By the involutive character of Matlis duality, it suffices to show that the type of $M$ does not exceed the value $r(R)(=r(A))$.

As $M=I / x I$, one has $\ell(M)=\operatorname{deg}(I)=\operatorname{deg}(R)=\ell(A)$. Further as $M$ is a faithful $A$-module, $\ell \ell(M)=\ell \ell(A)$. It follows then from lemma 4.6 that 


$$
\operatorname{type}(M) \leq 1+\operatorname{deg}(A)-\ell \ell(A) .
$$

Again from lemma 3.10, one obtains the inequality:

$$
\ell \ell(A) \leq 1+\operatorname{deg}(A)-r(A) \leq 4 .
$$

Inequality (1), gives the required bound, when $r(A)=1+\operatorname{deg}(A)-\ell \ell(A)$. Hence the result holds when $\ell \ell(A) \leq 2$ by lemma 4.7. When $r(A) \geq d-2$, lemma 4.6 indicates that either $\ell \ell(A) \leq 2$, or $r(A)=d-2=1+\operatorname{deg}(A)-$ $\ell \ell(A)$ and hence the result holds.

There remains but one further case:

$$
r(A)=\operatorname{deg}(A)-3 \quad \text { and } \quad \ell \ell(A)=3 .
$$

Suppose that there is a faithful $A$-module $M$ with $\operatorname{deg} M=\operatorname{deg} A$ and type $(M)=d-2$. Such an $M$ is (not-necessarly minimally) generated by $d-3$ elements of the socle and a non-socle element $z$. If $x \in(0: z)$ then $x \in(0: z) \cap(0:(0: M \mathfrak{m}))=(0: M)=0$. So $(0: z)=0$.

But since, $(0: z)=0$, there would be a injective $A$-morphism

$$
A \longrightarrow M \quad 1 \mapsto \alpha
$$

of modules of equal length, which must therfore be an isomophism. This contradicts the assumption on the type of $\mathrm{M}$.

REMARK 4.9. Combined with the Gulliksen result, this indicates that for one-dimensional Noetherian Cohen-Macaulay rings $R$ of multiplicity less than or equal to 6 , the minimal number of generators of a closed ideal is at most the Cohen-Macaulay type of $R$.

We close this section with some examples where the above result is applicable.

ExAmple 4.10. Let $k$ be a field. For some $n \in \mathrm{N}$, set

$$
R=k\left[\left[t^{n}, t^{n+1}, t^{n+2} \ldots, t^{2 n-1}\right]\right] .
$$

The ring $R$ is a one-dimensional Noetherian local ring of multiplicity $n$. The isomorphism class of the canonical module of $R$ is represented by the ideal $\left(t^{n}, t^{n+1}, t^{n+2}, t^{2 n-2}\right.$ ) (see [8]), hence the Cohen-Macaulay type of $R$ is $n-1$. The theorem implies that ever closed ideal of $R$ has at most $n-1$ generators.

ExAmple 4.11. Let $k$ be a field. Let $n \in \mathrm{N}$ with $n \geq 4$. Set

$$
R=k\left[\left[t^{n}, t^{n+1}, t^{n+2}, t^{2 n+5}, t^{2 n+6}, \ldots, t^{3 n-1}\right]\right] .
$$

The $\operatorname{ring} R$ is a one-dimensional Noetherian local ring of multiplicity $n$. The isomorphism class of the canonical module of $R$ is represented by the ideal 
$\left(t^{2 n}, t^{2 n+1}, \ldots, t^{3 n-4}\right)$ (see [8]), hence the Cohen-Maculay type of $R$ is $n-3$. The theorem implies that every closed ideal has at most $n-3$ generators.

\section{Residual faithfulness}

In this section we give a variation of the notion of closed ideals that is of interest primarily in the case Cohen-Macaulay ideals of codimension 1.

Let $R$ be a commutative ring, $M$ a finitely generated $R$-module and $I$ an ideal. There is an elementary relationship between the support of $M$ and the support $M / I M$ as expressed by their annihilators,

$$
\sqrt{I+\operatorname{Ann}(M)}=\sqrt{\operatorname{Ann}(M / I M)} .
$$

In general the more precise comparison between the annihilators is harder to make. To simplify the issue we assume that $M$ is faithful $R$-module and call it residually faithful with respect to the ideal $I$ if $\operatorname{Ann}(M / I M)=I$.

This occurs very often. It arises because $\operatorname{Ann}(M / I M)$ is an ideal integral over $I$ so coincides with it when $I$ is integrally closed. We are interested in the case when $I$ is generated by a system of parameters of a local ring and therefore will be almost always not be integrally closed.

Definition 5.1. Let $(R, \mathfrak{m})$ be a Cohen-Macaulay local ring of dimension $d$ and let $M$ be a faithful $R$-module. $M$ is called residually faithful if for some system of parameters $\mathbf{x}=x_{1}, \ldots, x_{d}$ generating the ideal $I$,

$$
\operatorname{Ann}(M / I M)=I \text {. }
$$

We are interested in studying this property in the class of Cohen-Macaulay modules, particularly those which are ideals (see also [11]). Note that without loss of generality we may assume that $R$ is complete, and hence has a canonical module $\omega$.

Proposition 5.2. A Cohen-Macaulay module $M$ is residually faithful if and only if the natural mapping

$$
\operatorname{Hom}_{R}(M, \omega) \otimes_{R} M \stackrel{\mathrm{ev}}{\longrightarrow} \omega
$$

is a surjection. In particular if $M$ is isomorphic to an ideal I then I is closed.

Proof. We used the argument earlier, specializing the mapping ev modulo a system of parameters $\mathrm{x}$ induces the corresponding evaluation mapping of $M /(\mathrm{x}) M$ : this is a consequence of

$$
\operatorname{Hom}_{R} /(\mathbf{x})(M /(\mathbf{x}) M, \omega /(\mathbf{x}) \omega) \simeq \operatorname{Hom}_{R}(M, \omega) \otimes_{R} R /(\mathbf{x}),
$$

valid for all maximal Cohen-Macaulay modules. The other assertion follows from Proposition 2.1. 
This shows for these modules the condition of being residually faithful is independent of the choice of the system of parameters.

Theorem 5.3. Let $(R, \mathfrak{m})$ be a Cohen-Macaulay local ring of type $r$. Then every residually faithful Cohen-Macaulay ideal I of height 1 has a reduction $J$ generated by $r$ elements. If the characteristic of the residue field is larger than $r$, the corresponding reduction number is at most $r-1$.

Proof. As in earlier arguments we write

$$
\sum \alpha(I)=\omega
$$

It follows from the Nakayama lemma that since $\omega$ is generated by $r$ elements, there are $r$ elements $a_{i} \in I$ and $r$ forms $\alpha_{i} \in \operatorname{Hom}_{R}(I, \omega)$ such that the elements $\alpha_{i}\left(a_{i}\right)$ generate $\omega$. We claim that $J=\left(a_{1}, \ldots, a_{r}\right)$ is a reduction of $I$ with the asserted properties.

For each $c \in I$ and each $\alpha_{j}$ we have a system of relations

$$
\alpha_{j}(c)=b_{1} j \alpha_{1}\left(a_{1}\right)+\cdots+b_{r j} \alpha_{r}\left(a_{r}\right) .
$$

For each element $z \in I$, we multiply all relations by $z$ and switch $z$ and $c$ (and each $a_{i}$ and $z$ ) within each $\alpha_{i}$ to get another set of relations

$$
c \cdot \alpha_{j}(z)=b_{1} j a_{1} \cdot \alpha_{1}(z)+\cdots+b_{r} j a_{r} \cdot \alpha_{r}(z) .
$$

We rewrite as a product of matrices

$$
\left[\begin{array}{ccc}
b_{11} a_{1}-c & \cdots & b_{1 r} a_{r} \\
\vdots & \ddots & \vdots \\
b_{r 1} a_{1} & \cdots & b_{r r} a_{r}-c
\end{array}\right] \cdot\left[\begin{array}{c}
\alpha_{1}(z) \\
\vdots \\
\alpha_{r}(z)
\end{array}\right]=0 .
$$

It follows that the determinant $f$ of the $r \times r$ matrix annihilates the submodule of $\omega$ generated by all $\alpha_{i}(z)$; but this is just $\omega$ itself. This means that

$$
0= \pm f=c^{r}+b_{1} c^{r-1}+\cdots+b_{r}, \quad b_{i} \in J^{i},
$$

which proves the first claim that $I$ is integral over $J$. The other assertion follows from a straightforward argument (see[13]).

REMARK 5.4. (i) It is not true, in dimension greater than 1 , that CohenMacaulay closed ideals are residually faithful. Consider the hypersurface ring $R=k[x, y, z] /\left(x^{2}+y^{2}-z^{2}\right)$ where $k$ is a field of characteristic $\neq 2$. $R$ is a normal ring and therefore every divisorial ideal is Cohen-Macaulay. The ideal $I=(x, y-z)$ is prime and a simple calculation shows that the annihilator of $I /(y, z) I$ is $(x, y, z) \neq(y, z)$. 
(ii) If $I$ is an ideal as in the Theorem above and $z_{1}, \ldots, z_{d-1}, d=\operatorname{dim} R$, is a system of parameters for $R / I$, the result of Proposition 3.9 implies that the number of generators of $\operatorname{Hom}_{R}(I, \omega)$ is precisely the number of irreducible components of $\left(I, z_{1}, \ldots, z_{d-1}\right)$.

(iii) It is a consequence of [3], [4] and [5] that regular local rings can be characterized as the Cohen-Macaulay local domains for which all nonzero ideals are residually faithful. This occurs for the following reason. Let $(R, \mathfrak{m})$ be a Cohen-Macaulay domain of dimension $d$ which is not a regular local ring and let $\mathrm{x}=\left(x_{1}, \ldots, x_{d}\right)$ be a system of parameters. According to [loc. cit.] the ideal $L=(\mathbf{x}): \mathfrak{m}$ satisfies $L^{2}=\mathrm{x} L$ so that the annihilator of $L / \mathrm{x} L$ is at least $L$, which properly contains $(\mathbf{x})$.

(iv) When the type of $R$ is at most 3, or is at least $\operatorname{deg}(R)-3$ the results of the previous section will imply that the number of generators of $I$ is bounded by the type of $R$, not just that it has a reduction with that number of elements. For this reason it would be very interesting to have closed ideals with larger than type of $R$ generators.

\section{REFERENCES}

1. H. Bass, On the ubiquity of Gorenstein rings, Math. Z. 82 (1963), 8-28.

2. W. Bruns and J. Herzog, Cohen-Macaulay Rings, Cambridge University Press, Cambridge, 1993.

3, A. Corso and C. Polini, Links of prime ideals and their Rees algebras J. Algebra 178 (1995), 224-238.

4. A. Corso, C. Polini, and W.V. Vasconcelos, Links of prime ideals, Math. Proc. Cambridge Philos. Soc. 115 (1994), 431-436.

5. S. Goto, Integral closedness of complete intersection ideals, J. Algebra 108 (1987), 151-160.

6. J. Herzog and E. Kunz, Der kanonische Modul eines Cohen-Macaulay Rings Lecture Notes in Math. 238, 1971.

7. T. H. Gulliksen, On the length of faithful modules over Artinian local rings, Math. Scand. 31 (1972), 78-82.

8. J. Jäger, Längeberechungen und kanonische Ideale in eindimensionalen Ringen, Arch. Math. (Basel) 29 (1977), 504-512.

9. J. Lipman, Stable ideals and Arf rings, Amer. J. Math. 93 (1971), 649-685.

10. H. Matsumura, Commutative Algebra, Benjamin/Cummings, Reading, Massachusetts, 1980.

11. W. V. Vasconcelos, Divisor Theory in Module Categories, North-Holland, Amsterdam, 1974.

12. W. V. Vasconcelos, Computing the integral closure of an affine domain, Proc. Amer. Math. Soc. 113 (1991), 633-638.

13. W. V. Vasconcelos, The reduction number of an algebra, Compositio Math. 104 (1996), 189197. 
14. R. Wiegand, Noetherian rings of bounded representation type, in Commutative Algebra (M. Hochster, C. Huneke and J. D. Sally, Eds.), MSRI Publications 15, Springer-Verlag, Berlin-Heidelberg-New York, 1989, 497-516.

DEPARTMENT OF MATHEMATICS NORTH DAKOTA STATE UNIVERSITY FARGO, N.D. 58105 USA
DEPARTMENT OF MATHEMATICS

RUTGERS UNIVERSITY

NEW BRUNSWICK, N. J. 08903

USA

Email: vasconce@rings.rutgers.edu 